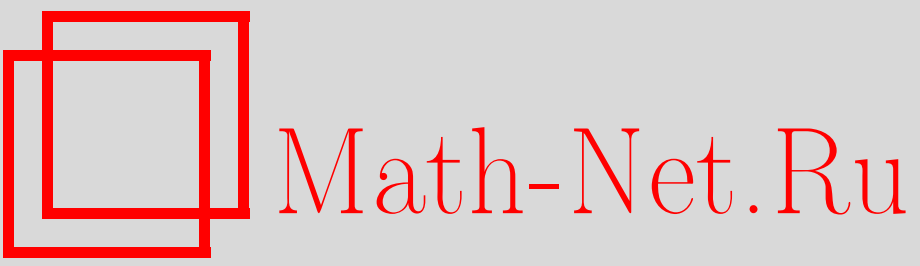

А. О. Барвинский, Д. В. Нестеров, Инфракрасная асимптотика ядра уравнения теплопроводности и нелокальное эффективное действие, ТМФ, 2005, том 143, номер 3, 328-356

DOI: https://doi.org/10.4213/tmf1817

Использование Общероссийского математического портала Math-Net.Ru подразумевает, что вы прочитали и согласны с пользовательским соглашением

http://www.mathnet.ru/rus/agreement

Параметры загрузки:

IP: 54.80 .73 .141

26 апреля 2023 г., 07:02:43 
ТЕОРЕТИЧЕСКАЯ

И МАТЕМАТИЧЕСКАЯ

ФИЗИКА

Том 143, № 3

июнь, 2005

\author{
(C) 2005 г. А.О. Барвинский*, Д. В. Нестеров*
}

\title{
ИНФРАКРАСНАЯ АСИМПТОТИКА ЯДРА УРАВНЕНИЯ ТЕПЛОПРОВОДНОСТИ И НЕЛОКАЛЬНОЕ ЭФФЕКТИВНОЕ ДЕЙСТВИЕ
}

Предлагается обзор новых результатов в непертурбативной теории ядра уравнения теплопроводности и его асимптотике "позднего" времени, описывающей инфракрасное поведение квантового эффективного действия для безмассовых теорий. В частности, выводится обобщение потенциала Коулмена-Вайнберга для теорий с неоднородным фоновым полем. При таком обобшении получается новое нелокальное и непертурбативное действие, описывающее эффекты в области перехода между внутренней частью пространства-времени и его бесконечностью. В четырехмерном пространстве эти эффекты приводят к делокализации логарифмического потенциала Коулмена-Вайнберга, в то время как в размерностях $d>4$ доминирующим оказывается вклад степенной нелокальной структуры, не зависящей от параметра перенормировки. Непертурбативное поведение ядра уравнения теплопроводности рассматривается также для асимптотически плоского искривленного пространства-времени. В частности, анализируются конформные свойства ядра уравнения теплопроводности для случая конформно-инвариантного скалярного поля и обсуждается проблема выделения эффективного космологического члена из нелокального эффективного действия.

Ключевые слова: эффективное действие, нелокальные теории поля, разложение Швингера-де Витта.

\section{1. ВВЕДЕНИЕ: ЯДРО УРАВНЕНИЯ ТЕПЛОПРОВОДНОСТИ И ЭФФЕКТИВНОЕ ДЕЙСТВИЕ}

В настоящее время широко признана важная роль нелокальных процессов в квантовой физике. В отличие от поляризационных эффектов в вакууме при низких энергиях в массивных теориях, они характеризуют высокоэнергетические квантовые эффекты в массивных теориях или инфракрасное поведение в безмассовых теориях. Даже в моделях с “хорошим низкоэнергетическим поведением", таких как эйнштейновская гравитация, нелокальные явления вызывают возрастающий интерес в связи с попытками разре-

Статья написана по заказу Редколлегии.

*Физический институт им. П. Н. Лебедева РАН, Москва, Россия. E-mail: barvin@lpi.ru, nesterov@lpi.ru 
шения проблем космологической постоянной и наблюдаемого космологического ускорения с помошью нелокальных модификаций теории для больших расстояний. Эти модификации обычно требуют непертурбативного рассмотрения ввиду непертурбативных аспектов проблемы Захарова-Ван Дамма-Велтмана [1] и наличия скрытого непертурбативного масштаба длины в гравитационных моделях с дополнительными измерениями [2].

С другой стороны, нелокальности также (и, более того, в основном) возникают в силу фундаментальных квантовых эффектов материальных полей или петлевых гравитационных эффектов, которые, например, могут играть важную роль в теории гравитационного излучения [3], [4] и космологии [5]. Эти механизмы возникновения нелокальностей являются альтернативой популярным феноменологическим механизмам инфракрасных модификаций, индуцированным, к примеру, бранными моделями с дополнительными измерениями [6], [7] или другими современными гравитационными сценариями [8]. Это делает непертурбативный анализ нелокальных квантовых эффектов интересным и многообешающим.

Основным инструментом для описания таких эффектов является формализм квантового эффективного действия и техника его вычисления с помошью ядра (уравнения) теплопроводности. Для теории поля обшего вида его классическое действие $S[\varphi]$ порождает обратньй пропагатор - оператор линейных полевых возмушений над фоновым полем $\varphi(x)$ :

$$
S[\varphi] \rightarrow F(\nabla) \delta(x, y)=\frac{\delta^{2} S[\varphi]}{\delta \varphi(x) \delta \varphi(y)} .
$$

Тогда квантовое эффективное действие $\Gamma[\varphi]$ следует из соответствуюшего классического действия как петлевое разложение по степеням $\hbar$ :

$$
S[\varphi] \rightarrow \Gamma=S[\varphi]+\hbar \Gamma_{1-\operatorname{loop}}[\varphi]+\hbar^{2} \Gamma_{2-\operatorname{loop}}[\varphi]+\cdots .
$$

Несколько первых порядков этого разложения графически представлены как фейнмановские диаграммы, в которых пропагатор - функция Грина оператора (1.1) - и соответствуюшие вершины вычислены на произвольном фоне $\varphi$,

$$
\left.\Gamma_{\text {1-loop }}=\frac{1}{2} \operatorname{Tr} \ln F(\nabla)=\frac{1}{2} \bigcirc, \quad \Gamma_{\text {2-loop }}=\frac{1}{8}\right\}+\frac{1}{12} \bigcirc .
$$

Однопетлевая часть (1.3) выделена в том смысле, что она не содержит явно вершин классического действия (до тех пор пока она не раскладывается по степеням фонового поля $\varphi$ ) и представима в виде функционального следа логарифма оператора $F(\nabla)$.

В локальных теориях поля в достаточно общем случае оператор (1.1) представим в виде

$$
F(\nabla)=\square-V(x),
$$

где $V(x)$ - некоторый потенциал, а $\square=g^{\mu \nu} \nabla_{\mu} \nabla_{\nu}$ - ковариантный даламбертиан в искривленном пространстве-времени с метрикой $g_{\mu \nu}$. 
Весьма эффективный путь анализа фейнмановских диаграмм типа (1.3) на произвольном фоне, т.е. с произвольными метрикой и потенциалом, основан на использовании ядра теплопроводности

$$
K(s \mid x, y)=\exp (s F(\nabla)) \delta(x, y)
$$

Оно удовлетворяет уравнению теплопроводности с единичным начальным условием при $s=0$,

$$
\frac{\partial K(s)}{\partial s}=F(\nabla) K(s), \quad K(0)=\mathbb{I},
$$

и посредством интегрирования по собственному времени позволяет вычислить основной элемент фейнмановской диаграммной техники - пропагатор оператора (1.1)

$$
G(x, y) \equiv \frac{1}{F(\nabla)} \delta(x, y)=-\int_{0}^{\infty} d s K(s \mid x, y)
$$

и в замкнутой форме - однопетлевое эффективное действие

$$
\begin{aligned}
\Gamma_{1 \text {-loop }} & =\frac{1}{2} \int_{0}^{\infty} \frac{d s}{s} \operatorname{Tr} K(s), \\
\operatorname{Tr} K(s) & =\int d x K(s \mid x, x) .
\end{aligned}
$$

Эффективность использования ядра теплопроводности и метода собственного времени основана на хорошо известном и универсальном поведении $K(s \mid x, y)$ для оператора второго порядка по производным общего вида (1.4) при $s \rightarrow 0$. Данньй предел определяет ультрафиолетовые расходимости и аномалии в теории поля, перенормировку и разложение по степеням производных, лежащих в основе вакуумных поляризационных эффектов.

Наоборот, нелокальные члены возникают из вклада верхнего предела в интеграле по собственному времени (1.8), что делает асимптотику “позднего" времени $\operatorname{Tr} K(s)$ существенной для другого класса эффектов, включающих рождение частиц и рассеяние ${ }^{1)}$. Подынтегральное выражение - след ядра теплопроводности, с учетом его асимптотики позднего времени впервые было изучено с помошью ковариантного нелокального разложения по кривизнам в [9], [11]-[13]. Цель данной работы - дать краткий обзор этих старых результатов, а также представить некоторые последние достижения в теории ядра теплопроводности, касающиеся непертурбативных асимптотик на плоском пространстве-времени [14], обобшения этих результатов на искривленное пространство-время [15], а также приложение их к теории нелокального эффективного действия.

\footnotetext{
1) В данной статье эффективное действие определяется в евклидовом пространстве с положительно-определенной метрикой. Его приложения в физическом пространстве-времени с лоренцевой сигнатурой основаны на методах аналитического продолжения, как, например, обычный виковский поворот в теории рассеяния (для in-out матричных элементов) или специальная прескрипция “запаздывания" в широком классе задач с фоновыми полями (вычисление in-in средних значений) [9], [10]. Эти методы нетривиально работают в применении к нелокальным объектам и включают как обычную теорию возмушений, так и ее частичные пересуммирования в соответствии с непертурбативной техникой, описанной в данной статье.
} 


\section{2. АППРОКСИМАЦИОННЫЕ СХЕМЫ И ИНФРАКРАСНОЕ ПОВЕДЕНИЕ}

$\mathrm{C}$ целью упрощения изложения в этом разделе мы будем работать в плоском пространстве-времени с метрикой $g_{\mu \nu}=\delta_{\mu \nu}$ и только в случае необходимости кратко упомянем соответствуюшие изменения, учитываюшие кривизну. Вместе с этим будет рассматриваться случай скалярного поля, где ядро теплопроводности (1.5) есть бискалярный объект без индексов (как спиновых, так и изотопических). В этом случае поведение ядра теплопроводности при малом (собственном) времени, ответственное за перенормировку в локальной квантовой теории поля, выглядит наиболее просто:

$$
\begin{gathered}
K(s \mid x, y)=\frac{1}{(4 \pi s)^{d / 2}} \exp \left\{-\frac{|x-y|^{2}}{4 s}\right\} \Omega(s \mid x, y), \\
\Omega(s \mid x, y) \rightarrow 1, \quad s \rightarrow 0
\end{gathered}
$$

где $d$ есть размерность пространства-времени. Этот квазиклассический анзац для ядра теплопроводности гарантирует стремление к $\delta$-функции $\delta(x, y)$ при $s \rightarrow 0$ и содержит всю нетривиальную информацию о потенциале $V(x)$ в функции $\Omega(s \mid x, y)$, которая аналитична при $s=0$. Ее разложение по степеням $s$ лежит в основе техники локального разложения Швингера-де Витта.

2.1. Техника локального разложения Швингера-де Витта. Из уравнения теплопроводности (1.6) легко выводится набор рекуррентных уравнений для коэффициентов разложения $\Omega(s \mid x, y)$ по малому параметру собственного времени,

$$
\Omega(s \mid x, y)=\sum_{n=0}^{\infty} a_{n}(x, y) s^{n}, \quad s \rightarrow 0 .
$$

Коэффициенты этого разложения играют важную роль в квантовой теории поля и в физической литературе чаще всего называются коэффициентами Швингера-де Витта. Уравнения для $a_{n}(x, y)$ могут быть в замкнутой форме решены для пределов совпадения $a_{n}(x, x)$ в терминах потенциала $V(x)$ и его производных. Для оператора (1.4) несколько первых коэффициентов разложения при совпадающих аргументах выглядят следуюшим образом:

$$
\begin{gathered}
a_{0}(x, x)=1, \\
a_{1}(x, x)=-V(x)+\frac{1}{6} R(x), \\
a_{2}(x, x)=\frac{1}{2} V^{2}(x)+\frac{1}{6} \square V(x)-\frac{1}{6} R(x) V(x)+O\left(R_{\mu \nu \alpha \beta}^{2}\right),
\end{gathered}
$$

где мы учли вклад скаляра кривизны пространства-времени и символически обозначили квадратичньй вклад тензора кривизны во второй коэффициент. Явно видно, что все эти величины являются локальными функциями коэффициентов исходного оператора и их производных. Размерность $a_{n}(x, x)$ в единицах обратной длины растет с ростом $n$ и составляется из степеней размерных величин $V(x), R_{\mu \nu \alpha \beta}$ и их производных. 
Предположим теперь, что вместо теории с оператором (1.4) мы рассматриваем теорию массивного поля с большой массой $m$. Это соответствует замене исходного оператора

$$
F(\nabla) \rightarrow F(\nabla)-m^{2} .
$$

Соответствуюшее ядро теплопроводности (2.1) при такой замене, очевидно, приобретает общий экспоненциальный фактор $\exp \left(-s m^{2}\right)$, подавляющий вклад больших значений $s$ в интеграле по собственному времени (1.8),

$$
K(s \mid x, y)=\frac{1}{(4 \pi s)^{d / 2}} \exp \left\{-\frac{|x-y|^{2}}{4 s}-s m^{2}\right\} \Omega(s \mid x, y) .
$$

Подставляя это выражение с учетом разложения (2.3) в (1.8) и почленно интегрируя получившийся ряд, мы находим однопетлевое эффективное действие в виде асимптотического $1 / m^{2}$-разложения [11], [14], [16], [17]:

$$
\begin{aligned}
& \frac{1}{2} \operatorname{Tr} \ln {\left[F(\nabla)-m^{2}\right]=-\frac{1}{2(4 \pi)^{d / 2}} \int d x \int_{0}^{\infty} \frac{d s}{s^{d / 2+1}} e^{-s m^{2}} \sum_{n=0}^{\infty} s^{n} a_{n}(x, x)=} \\
&=\Gamma_{\text {div }}+\Gamma_{\log }-\frac{1}{2}\left(\frac{m^{2}}{4 \pi}\right)^{d / 2} \int d x \sum_{n=d / 2+1}^{\infty} \frac{\Gamma(n-d / 2)}{\left(m^{2}\right)^{n}} a_{n}(x, x) .
\end{aligned}
$$

Первые $d / 2$ интегралов (мы предполагаем, что $d$ четно) расходятся в пределе малых $s$, тем самым генерируя ультрафиолетовые расходимости $\Gamma_{\mathrm{div}}$, которые определяются первыми $d / 2$ коэффициентами Швингера-де Витта. При размерной регуляризации

$$
\Gamma_{\mathrm{div}}=\frac{1}{2(4 \pi)^{d / 2}} \int d x \sum_{n=0}^{d / 2}\left[\frac{1}{\omega-d / 2}-\Gamma^{\prime}\left(\frac{d}{2}-n+1\right)\right] \frac{\left(-m^{2}\right)^{d / 2-n}}{(d / 2-n) !} a_{n}(x, x),
$$

где $2 \omega$ - параметр продолжения по размерности.

Логарифмические расходимости описываются логарифмическим членом,

$$
\Gamma_{\log }=\frac{1}{2(4 \pi)^{d / 2}} \int d x \sum_{n=0}^{d / 2} \frac{\left(-m^{2}\right)^{d / 2-n}}{(d / 2-n) !} \ln \frac{m^{2}}{\mu^{2}} a_{n}(x, x),
$$

содержашим параметр перенормировки массы $\mu^{2}$, отражаюший перенормировочный произвол.

В таком представлении каждое конечное слагаемое в правой части действия (2.7) локально, но эта локальность имеет место только в области применимости разложения, когда масса велика и слагаемые в асимптотическом ряду быстро убывают с ростом $n$. Это условие выполняется, когда параметр массы $m$ велик по сравнению как с потенциалом, так и с его производными:

$$
1 \gg \frac{a_{n}}{\left(m^{2}\right)^{n}} \sim\left(\frac{V}{m^{2}}\right)^{n}, \quad\left(\frac{\nabla}{m}\right)^{k}\left(\frac{V}{m^{2}}\right)^{l}, \quad k+2 l=2 n .
$$


В присутствии гравитационного поля эти ограничения также включают в себя условия малости кривизны пространства-времени и его ковариантных производных по сравнению с параметром массы. Таким образом, локальное разложение Швингера-де Витта применимо только для медленно меняющихся полей малой амплитуды по сравнению с массовым масштабом рассматриваемой модели.

Для полей больших напряженностей или быстро меняюшихся полей разложение Швингера-де Витта становится неприменимым и полностью рушится в безмассовом пределе $m \rightarrow 0$. Возникает естественный вопрос, какова структура эффективного действия в такой ситуации и как его вычислять. Далее мы рассмотрим два пертурбативных метода, которые совершенствуют технику Швингера-де Витта, распространяя ее на класс безмассовых моделей, и затем в разделе 3 перейдем к непертурбативной технике, основанной на непертурбативной асимптотике позднего времени ядра теплопроводности

2.2. Модифицированное разложение Швингера-де Витта. Первый метод может быть назван модифицированным разложением Швингера-де Витта, в силу того что он основан на пересуммировании исходного разложения при замене массового члена на потенциал $V(x)$ в операторе $(2.5), m^{2} \rightarrow V(x)$. Когда потенциал положительно определен (это будет предполагаться в дальнейшем), он может сыграть роль подавляюшего фактора (при больших временах) по аналогии с массовым членом в интеграле (2.7). Для этого необходимо выделить экспоненциальную зависимость от $s V(x)$ в $\Omega(s \mid x, y)$ : $\Omega(s \mid x, y)=e^{-s V(x)} \widetilde{\Omega}(s \mid x, y)$, и вместо (2.6) написать анзац

$$
K(s \mid x, y)=\frac{1}{(4 \pi s)^{d / 2}} \exp \left\{-\frac{|x-y|^{2}}{4 s}-s V(x)\right\} \widetilde{\Omega}(s \mid x, y),
$$

где новая функция, отмеченная тильдой, разлагается по степеням $s$ с модифицированными коэффициентами Швингера-де Витта:

$$
\widetilde{\Omega}(s \mid x, y)=\sum_{n=0}^{\infty} \tilde{a}_{n}(x, y) s^{n} .
$$

Очевидно, этому разложению отвечает частичное пересуммирование в исходном ряду (2.3) слагаемых, содержащих только степени недифференцированного потенциала. Новые коэффициенты $\tilde{a}_{n}(x, x)$, по сравнению с коэффициентами в исходном разложении, формируются из меньшего числа слагаемых и не содержат членов с недифференцированным потенциалом (таким образом, все члены ряда, кроме $\tilde{a}_{0}=1$, в отсутствие гравитации становятся равными нулю при $\nabla V=0)$. Коэффициенты исходного разложения Швингера-де Витта могут быть выражены через новые как конечные полиномы по $V$ с коэффициентами, построенными из градиентов потенциала,

$$
a_{m}(x, x)=\frac{(-V)^{m}}{m !}+\sum_{n=1}^{d / 2} \frac{(-V)^{m-n}}{(m-n) !} \tilde{a}_{n}(x, x)=\frac{(-V)^{m}}{m !}+O(\nabla V)
$$


Теперь интеграл по собственному времени в (2.7) даже при $m^{2}=0$ имеет инфракрасное обрезание при $s \sim 1 / V(x)$, и в этом случае эффективное действие аналогично $(2.7) \mathrm{c}$ заменой $m^{2}$ на $V(x)$, а $a_{n}(x, x)$ на $\tilde{a}_{n}(x, x)$ :

$$
\sum_{n} \frac{a_{n}(x, x)}{\left(m^{2}\right)^{n}} \rightarrow \sum_{n} \frac{\tilde{a}_{n}(x, x)}{V^{n}(x)} .
$$

В частности, ультрафиолетовые расходимости определяются безмассовым пределом $\Gamma_{\text {div }}$, а логарифмическая часть порож дает ( $d$-мерное) слагаемое Коулмена-Вайнберга $\Gamma_{\log } \rightarrow \Gamma_{\mathrm{CW}}+O(\nabla V)$,

$$
\Gamma_{\mathrm{CW}}=\frac{1}{2(4 \pi)^{d / 2}} \int d x \frac{(-V)^{d / 2}(x)}{(d / 2) !} \ln \frac{V(x)}{\mu^{2}},
$$

с поправками, зависяшими от производных потенциала (ср. с формулой (2.13) при $m=d / 2) . \quad \Gamma_{\mathrm{CW}}$ есть интеграл по пространству-времени от эффективного потенциала Коулмена-Вайнберга. Например, в четырехмерии в модели $\varphi^{4}$ самодействуюшего скалярного поля с $V(\varphi) \sim \varphi^{2}$ этот эффективный потенциал имеет хорошо известный вид $\varphi^{4} \ln \left(\varphi^{2} / \mu^{2}\right) / 64 \pi^{2}$.

Из (2.14) следует, что, в отличие от исходного разложения Швингера-де Витта, его модифицированная версия представляет собой разложение по производным от $V$, а не по степеням самого $V$. Действительно, коэффициенты модифицированного ряда не содержат слагаемых с недифференцированным потенциалом, и типичная структура слагаемых, входящих в $\tilde{a}_{n}(x, x)$, представляется $m$ производными, действуюшими разными способами на произведение $j$ потенциалов: $\nabla^{m} V^{j}(x)$, где $m+2 j=2 n$. Оказывается,

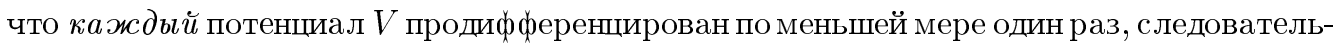
но $m \geqslant j$. Поэтому типичное слагаемое в разложении (2.14) может быть символически записано как

$$
\frac{\tilde{a}_{n}}{V^{n}} \sim \sum_{j=1}^{[2 n / 3]} \frac{\nabla^{2 n-2 j} V^{j}}{V^{n}}
$$

где верхний предел $j$ есть целая часть $2 n / 3$. Таким образом, модифицированное разложение оказывается эффективным, пока градиент потенциала остается меньше самого потенциала,

$$
\frac{\nabla^{2} V(x)}{V^{2}(x)} \ll 1, \quad \frac{(\nabla V(x))^{2}}{V^{3}(x)} \ll 1, \quad \ldots .
$$

Когда потенциал ограничен снизу большой положительной константой, эти условия, очевидно, оказываются выполненными во всем пространстве-времени. Однако этот случай не представляет интереса, поскольку он лишь воспроизводит исходное разложение Швингера-де Витта с массовым членом $m^{2}$, играющим роль нижней границы потенциала. Больший интерес представляет случай асимптотически пустого пространства, когда потенциал и его производные стремятся к нулю по степенному закону

$$
V(x) \sim \frac{1}{|x|^{p}}, \quad \nabla^{m} V(x) \sim \frac{1}{|x|^{p+m}}, \quad|x| \rightarrow \infty,
$$


для некоторого положительного $p$. Для случая такого потенциала слагаемые ряда теории возмушений (2.14) обладают следуюшим поведением:

$$
\frac{\tilde{a}_{n}(x, x)}{V^{n}(x)} \sim \sum_{j=1}^{[2 n / 3]}|x|^{(p-2)(n-j)},
$$

и убывают с ростом $n$, только если $p<2$. Для $p \geqslant 2$ модифицированное разложение по производным потенциала полностью теряет смысл. Таким образом, это разложение хорошо определено для медленно убывающих потенциалов вида (2.18) с $p<2$. В этом случае потенциал $V(x)$ не интегрируем по всему пространству-времени $\left(\int d x V(x)=\infty\right)$ и, более того, выражение $(1 / \square) V(x)$ не является хорошо определенным ${ }^{2)}$. Поэтому полученные условия на потенциал слишком ограничительны и не позволяют описать физически интересные ситуации, в которых параметр $p$ зачастую совпадает с размерностью пространства-времени $d$. Кроме того, модифишированное разложение полностью локально и не позволяет уловить нелокальные слагаемые в эффективном действии.

Таким образом, требуется альтернативная техника для исследования вклада позднего времени в интеграл по собственному времени, в частности, чтобы понять, определен ли (и если да, то при каких условиях) сам интеграл для безмассовых теорий. Ответ на этот вопрос может дать изучение асимптотики позднего времени $(s \rightarrow \infty)$ ядра теплопроводности, которая может быть пертурбативно проанализирована в рамках ковариантной теории возмушений [9], [11]-[13], [18].

2.3. Ковариантная теория возмущений. В ковариантной теории возмушений сам потенциал рассматривается как возмущение, и решение уравнения теплопроводности находится в виде ряда по степеням потенциала. C точки зрения разложения Швингера-де Витта, такое рассмотрение соответствует бесконечному пересуммированию всех слагаемых с данной степенью потенциала и произвольным количеством производных. Результат представим в виде

$$
\operatorname{Tr} K(s) \equiv \int d x K(s \mid x, x)=\sum_{n=0}^{\infty} \operatorname{Tr} K_{n}(s),
$$

где

$$
\operatorname{Tr} K_{n}(s)=\int d x_{1} d x_{2} \ldots d x_{n} F_{n}\left(s \mid x_{1}, x_{2}, \ldots, x_{n}\right) V\left(x_{1}\right) V\left(x_{2}\right) \ldots V\left(x_{n}\right) .
$$

Нелокальные формфакторы $F_{n}\left(s \mid x_{1}, x_{2}, \ldots, x_{n}\right)$ были явно вычислены в [9], [11], [12] до $n=3$ включительно. В присутствии калибровочных полей и гравитации это разложение может быть легко обобщено путем включения кривизн расслоенного пространства $\mathcal{R}_{\mu \nu}$ и тензора Риччи ${ }^{3)}$ в полный набор пертурбативных полевых напряженностей

\footnotetext{
2) Для сходимости интеграла в выражении $(1 / \square) V(x)$ потенциал $V(x)$ должен убывать по меньшей мере как $1 /|x|^{3}$ для пространства-времени любой размерности [11].

${ }^{3)}$ В асимптотически плоском пространстве-времени с естественными вакуумными граничными условиями тензор кривизны Римана-Кристоффеля может быть выражен по теории возмущений в терминах тензора Риччи [12], [18]. Именно поэтому он не входит в разложение как независимая величина.
} 
$V \rightarrow \mathcal{R}=\left(V, \mathcal{R}_{\mu \nu}, R_{\mu \nu}\right)$ и ковариантизации соответствующих нелокальных формфакторов.

В [11] было показано, что при $s \rightarrow \infty$ члены разложения ведут себя как

$$
\operatorname{Tr} K_{n}(s)=O\left(\frac{1}{s^{d / 2-1}}\right), \quad n \geqslant 1
$$

и, следовательно, в размерности $d \geqslant 3$ интеграл в (1.8) сходится в инфракрасном пределе,

$$
\Gamma \sim \int^{\infty} \frac{d s}{s} O\left(\frac{1}{s^{d / 2-1}}\right)<\infty .
$$

В размерностях $d=1$ и $d=2$ такого разложения для Г не сушествует, за исключением особого случая безмассовой теории в искривленном двумерном пространстве,где оно воспроизводит действие Полякова [11], [19], [20], которое также может быть получено путем интегрирования конформной аномалии [19], [20],

$$
\Gamma_{\Pi} \sim \int d^{2} x g^{1 / 2} R \frac{1}{\square} R
$$

Ковариантная теория возмушений применима для $d \geqslant 3$ и достаточно малого потенциала $V$, так что получаюшееся эффективное действие обладает свойством аналитичности по потенциалу при $V=0$. Таким образом, серьезный недостаток такого подхода в том, что он не позволяет преодолеть рамки теории возмушений и, в частности, не позволяет установить неаналитические (по потенциалу) структуры в действии, если таковые присутствуют.

\section{3. НЕПЕРТУРБАТИВНАЯ АСИМПТОТИКА ПОЗДНЕГО ВРЕМЕНИ}

Непертурбативная техника для ядра теплопроводности основана на приближении, качественно отличном от использованных в предыдущем разделе. Вместо наложения тех или иных условий малости на фоновые поля, мы рассмотрим довольно обший случай, но рассмотрим предел большого собственного времени $s \rightarrow \infty$. Продолжая работать в плоском пространстве-времени с метрикой $g_{\mu \nu}=\delta_{\mu \nu}$, подставим анзац $(2.1)$ в уравнение теплопроводности, откуда получим уравнение на неизвестную функцию $\Omega(s \mid x, y)$

$$
\frac{\partial \Omega}{\partial s}+\frac{1}{s}(x-y)^{\mu} \nabla_{\mu} \Omega=F(\nabla) \Omega
$$

Далее, предположим сушествование следуюшего $1 / s$-разложения для этой функции, которое вытекает, в частности, из ковариантной пертурбативной теории для $K(s \mid x, y)$ [11], [14] (она была вкратце описана выше - в ней отсутствовали неаналитические по $1 / s$ слагаемые типа $\ln (1 / s))$ :

$$
\Omega(s \mid x, y)=\Omega_{0}(x, y)+\frac{1}{s} \Omega_{1}(x, y)+O\left(\frac{1}{s^{2}}\right)
$$


и выведем совокупность рекуррентных уравнений на коэффициенты этого разложения

$$
\begin{aligned}
& F(\nabla) \Omega_{0}(x, y)=0 \\
& F(\nabla) \Omega_{1}(x, y)=(x-y)^{\mu} \nabla_{\mu} \Omega_{0}(x, y), \\
& \ldots \ldots \ldots \ldots \ldots \ldots \ldots \ldots \ldots \ldots
\end{aligned}
$$

Очевидная трудность с выбором конкретного решения для этой цепочки уравнений состоит в отсутствии хорошо поставленной граничной задачи. Дело в том, что естественные нулевые граничные условия на бесконечности для исходного ядра $K(s \mid x, y)$ не налагают каких-либо граничных условий на функцию $\Omega(s \mid x, y)$, кроме ограничения на рост $\Omega(s \mid x, y)$ медленнее чем $\exp \left[+|x-y|^{2} /(2 s)\right]$, из-за экспоненциального фактора в (2.1). С другой стороны, имеющаяся свобода в выборе неубывающих при $|x| \rightarrow \infty$ решений сушественно помогает найти эти самые решения, так как в противном случае нетривиальные решения могли бы вообще не существовать. В самом деле, для эллиптического уравнения (3.3) с положительно-определенным оператором $F(\nabla)$ (мы везде предполагаем положительную определенность оператора) ненулевые решения, убывающие на бесконечности, отсутствуют. Таким образом, единственный оставшийся критерий выбора решений для (3.3), (3.4) - это требование симметрии коэффициентов $\left(\Omega_{0}(x, y), \Omega_{1}(x, y), \ldots\right)$ относительно перестановки аргументов. Как будет показано далее, данный критерий, совместно с другими естественными предположениями, позволяет найти решения, которые потом будут проверяться на согласованность различными методами, включая теорию возмушений, вариационные уравнения на след ядра теплопроводности, в том числе по метрике, и т.д.

Отсутствие конкретных условий на убывание коэффициентов разложения (3.2) приводит еше к одной интересной особенности разложения при больших временах. Как будет показано, разложение функционального следа ядра теплопроводности, соответствуюшего (3.2), есть

$$
\operatorname{Tr} K(s)=\frac{1}{(4 \pi s)^{d / 2}}\left\{s W_{0}+W_{1}+O\left(\frac{1}{s}\right)\right\},
$$

что, очевидно, подразумевает, что, в отличие от определения (1.9), в этой формуле $W_{n} \neq$ $\int d x \Omega_{n}(x, x), n=0,1, \ldots$, из-за сдвига на единицу в степенях $s$. Объясняется это кажущееся несоответствие между (3.2) и (3.5) тем, что $1 / s$-разложение (3.2) является неравномерным по аргументам $x$ и $y$ величины $\Omega(s \mid x, y)$. Следовательно, при фиксированном $s$ выражение $\Omega(s \mid x, x)$, взятое из разложения, становится некорректным и, как следствие, функциональный след ядра теплопроводности (требуюший интегрирования вплоть до бесконечности) не может быть получен путем наивного применения (1.9) к (3.2).

К счастью, есть способ преодолеть это затруднение. Функциональный след ядра теплопроводности может быть восстановлен по своему разложению с помошью решения следуюшего вариационного уравнения:

$$
\frac{\delta \operatorname{Tr} K(s)}{\delta V(x)}=-s K(s \mid x, x)
$$

2 Теоретическая и математическая физика, т. 143, № 3, 2005 г. 
которое является прямым следствием определения ядра теплопроводности. Присутствуюшее в правой части этого уравнения собственное время $s$ в первой степени объясняет, в частности, его дополнительную степень в (3.5) по сравнению с (3.2). Уравнение (3.6) распадается на последовательность вариационных уравнений

$$
\frac{\delta W_{n}}{\delta V(x)}=-\Omega_{n}(x, x), \quad n=0,1, \ldots,
$$

которые будут использоваться для вывода первых двух коэффициентов в (3.5).

3.1. Старший порядок разложения. Применим обозначенную выше стратегию вычисления для нахождения лидируюшего порядка в $1 / s$-разложении [14]. Сделаем естественное предположение, что $\Omega_{0}(x, y)$ при $|x| \rightarrow \infty$ не возрастает, не зависит от направления $n^{\mu}=x^{\mu} /|x|$ и соответственно стремится к некоторой конечной величине $C(y)$, зависящей только от $y$. Тогда решение (3.3), удовлетворяющее граничному условию $\left.\Omega_{0}(x, y)\right|_{|x| \rightarrow \infty}=C(y)$, единственно и может быть представлено в виде $\Omega_{0}(x, y)=$ $\Phi(x) C(y)$, где $\Phi(x)$ - функция специального вида:

$$
\Phi(x)=1+\frac{1}{\square-V} V(x) \equiv 1+\int d y G(x, y) V(y),
$$

удовлетворяюшая однородному уравнению с единичным граничным условием на бесконечности

$$
F(\nabla) \Phi(x)=0, \quad \Phi(x) \rightarrow 1, \quad|x| \rightarrow \infty .
$$

Из требования симметрии по $x$ и $y$ получаем, что $\Omega_{0}(x, y)=C \Phi(x) \Phi(y)$, где значение нормировочной константы $C=1$ следует из сопоставления с известным явным выражением для ядра теплопроводности в плоском пространстве с нулевым потенциалом $V(x)=0$. Таким образом,

$$
\Omega_{0}(x, y)=\Phi(x) \Phi(y) .
$$

Заметим, что если наивно проинтегрировать данное выражение по всему пространству, то след ядра теплопроводности оказывается плохо определенным, из-за того что интеграл от величины $\Omega_{0}(x, y)$ при совпадающих аргументах расходится,

$$
\int d x \Omega(s \mid x, x)=\int d x \Phi^{2}(x)=\infty .
$$

Наоборот, вариационное уравнение (3.7) на функционал $W_{0}$ удовлетворяет условию совместности в силу соотношения

$$
\frac{\delta \Phi(x)}{\delta V(y)}=G(x, y) \Phi(y)
$$

и имеет следующее решение в виде хорошо определенного сходящегося интеграла [14]:

$$
W_{0}=-\int d x V(x) \Phi(x) .
$$


3.2. Сублидирующий порядок. Для сублидирующего порядка уравнение (3.4) принимает вид

$$
F(\nabla) \Omega_{1}(x, y)=(x-y)^{\mu} \nabla_{\mu} \Phi(x) \Phi(y)
$$

с неоднородным слагаемым в правой части. Симметричное по $x$ и $y$ решение, вычисленное в [15],

$$
\begin{aligned}
\Omega_{1}(x, y)= & \frac{1}{\square_{x}-V_{x}}(x-y)^{\mu} \nabla_{\mu} \Phi(x) \Phi(y)+(x \leftrightarrow y)+ \\
& +2 \frac{1}{\square_{x}-V_{x}} \nabla_{\mu} \Phi(x) \frac{1}{\square_{y}-V_{y}} \nabla^{\mu} \Phi(y)
\end{aligned}
$$

не убывает на бесконечности ${ }^{4}$. Более того, в этом решении присутствуют линейно растушие слагаемые как по $x$, так и по $y$, что является следствием отсутствия убывания в правой части (3.13). Поэтому решение, вообше говоря, не единственно, но правильность данного выбора подкрепляется тем фактом, что соответствуюший предел совпадающих аргументов $\Omega_{1}(x, x)$ удовлетворяет условиям совместности вариационного уравнения (3.7) для $W_{1}[15]$ и совпадает с результатом непосредственного суммирования рядов теории возмушений для $\operatorname{Tr} K(s)$ [14]. Ответ для $W_{1}$ принимает вид

$$
W_{1}=\int d x\left\{1-2 \nabla_{\mu} \Phi \frac{1}{\square-V} \nabla^{\mu} \Phi\right\}
$$

где нетривиальное нелокальное слагаемое, квадратичное по градиентам $\Phi(x)$, задается хорошо определенным сходящимся интегралом, в отличие от первого слагаемого, которое добавлено как постоянная функционального интегрирования из сравнения полученного результата с точно известным решением для случая нулевого потенциала.

Для обоснования непертурбативных алгоритмов этого раздела и, в частности, для проверки правильности выбора решений для коэффициентов $1 / s$-разложения $(3.2)$ можно сравнить полученные результаты с результатами пересуммирования нелокальных рядов в пертурбативной технике [9], [11], [12]. В рамках этой техники след ядра теплопроводности разлагается в нелокальный ряд (2.20), (2.21) по степеням потенциала $V$ с вычислимыми явно коэффициентами - нелокальными формфакторами $F_{n}\left(s \mid x_{1}, x_{2}, \ldots, x_{n}\right)$. Их лидируюшее асимптотическое поведение при больших $s$ было получено в [11] и может быть распространено на первый сублидируюший порядок по $1 / s[14]$. В этой аппроксимации также можно явно просуммировать бесконечные ряды по степеням потенциала [14], [21], чтобы подтвердить полученные ранее ответы для $W_{0}(3.12)$ и $W_{1}(3.15)$.

\footnotetext{
4) Здесь и далее индекс дифференциального оператора в знаменателе обозначает аргумент, на который действует соответствующая функция Грина.
} 


\section{4. НОВОЕ НЕЛОКАЛЬНОЕ ЭФФЕКТИВНОЕ ДЕЙСТВИЕ}

Непертурбативная асимптотика ядра теплопроводности позволяет существенно улучшить вычисление эффективного действия. Новое приближение, которое учитывает поведение $\operatorname{Tr} K(s)$ как при малых, так и при больших временах, одновременно описывает и ультрафиолетовые, и инфракрасные свойства теории и генерирует новые структуры в эффективном действии. Эти структуры обобщают локальный потенциал Коулмена-Вайнберга (2.15) на случай, когда (фоновое) поле не однородно на всем пространстве-времени и стремится к нулю на бесконечности. Как было показано в п. 2.2 , только случай чрезвычайно медленного и поэтому физически не интересного убывания потенциала (2.18) с $p<2$ может быть описан по теории возмушений. Для случая более быстрого убывания при $|x| \rightarrow \infty$ инфракрасное обрезание интеграла по собственному времени в формализме модифицированного градиентного разложения перестает работать, поэтому приходится использовать или нелокальную теорию возмушений п. 2.3 , или непертурбативную асимптотику раздела 3 . Последняя позволяет получить нелокальное непертурбативное действие, которое нетривиально учитывает явления взаимодействия меж ду внутренней частью пространства-времени при конечных $|x|$ и областью $|x| \rightarrow \infty$, где потенциал стремится к нулю.

При построении нового приближения ключевая идея заключается в замене $\operatorname{Tr} K(s)$ в (1.8) на некоторый приближенный функционал $\operatorname{Tr} \bar{K}(s)$ такой, что его асимптотики малого и позднего времен совпадают с асимптотиками точного функционала, а интеграл по $s$ вычисляется явно. Здесь мы используем простейшую возможность, а именно: возьмем две простые функции $\operatorname{Tr} K_{<}(s)$ и $\operatorname{Tr} K_{>}(s)$,

$$
\begin{aligned}
& \operatorname{Tr} K_{<}(s)=\frac{1}{(4 \pi s)^{2}} \int d x e^{-s V}, \\
& \operatorname{Tr} K_{>}(s)=\frac{1}{(4 \pi s)^{2}} \int d x(1-s V \Phi),
\end{aligned}
$$

которые просто являются асимптотиками $\operatorname{Tr} K(s)$ при $s \rightarrow 0$ (модифицированное градиентное разложение с опущенными слагаемыми, содержашими производные от потенциала) и $s \rightarrow \infty$, и используем их для аппроксимации $\operatorname{Tr} K(s)$ соответственно в областях $0 \leqslant s \leqslant s_{*}$ и $s_{*} \leqslant s<\infty$ для некоторого $s_{*}$ :

$$
\operatorname{Tr} \bar{K}(s)= \begin{cases}\operatorname{Tr} K_{<}(s), & s<s_{*}, \\ \operatorname{Tr} K_{>}(s), & s>s_{*} .\end{cases}
$$

Значение $s_{*}$ будет вычисляться из требования совпадения двух функций при $s_{*}$, что будет гарантировать стационарность $\bar{\Gamma}$ относительно выбора $s_{*}, \partial \bar{\Gamma} / \partial s_{*}=0$,

$$
\operatorname{Tr} K_{<}\left(s_{*}\right)=\operatorname{Tr} K_{>}\left(s_{*}\right) .
$$

Таким образом, новое приближение для эффективного действия можно записать в виде

$$
\bar{\Gamma}=-\frac{1}{2} \int_{0}^{s_{*}} \frac{d s}{s} \operatorname{Tr} K_{<}(s)-\frac{1}{2} \int_{s_{*}}^{\infty} \frac{d s}{s} \operatorname{Tr} K_{>}(s),
$$


а его отклонение от точного $\Gamma_{1 \text {-loор }}$, пропорциональное $\operatorname{Tr}[K(s)-\bar{K}(s)]$, можно рассматривать как возмушение. Такая кусочно-гладкая аппроксимация эффективно работает, только если области применимости двух асимптотических разложений (для малых и больших $s$ ) перекрываются и точка $s_{*}$ попадает в область перекрытия. В этом случае поправки по отклонению $\operatorname{Tr} \bar{K}(s)$ от точного $\operatorname{Tr} K(s)$ равномерно ограничены всюду и можно ожидать, что (4.5) даст хорошее приближение к точному результату. Такое требование может выполняться по меньшей мере для двух достаточно широких классов потенциалов $V(x)$. Для простоты предположим, что потенщиал обладает конечной амплитудой внутри компактного носителя с характерным масштабом $R$ [14],

$$
\begin{aligned}
V(x) & =0, & & |x|>R, \\
V(x) & \sim V_{0}, & & |x|<R,
\end{aligned}
$$

и его производные не слишком большие и равномерно ограничены величиной порядка $V_{0} / R$.

4.1. Малый потенциал. Первому классу принадлежат потенциалы, малые в единишах обратного масштаба длины компактного носителя,

$$
V_{0} R^{2} \ll 1
$$

Рассмотрим для начала случай четырехмерного пространства-времени, $d=4$. Несложные вычисления дают следуюший ответ [14], [21] для конечной части действия, справедливый с точностью до поправок, пропорциональных параметру малости (4.7):

$$
\begin{aligned}
\bar{\Gamma} & \frac{1}{64 \pi^{2}} \int d^{4} x V^{2}(x) \ln \left(\int d^{4} y V^{2}(y)\right)- \\
& -\frac{1}{64 \pi^{2}} \int d^{4} x V^{2}(x) \ln \left(\int d^{4} y V \frac{\mu^{2}}{V-\square} V(y)\right), \quad d=4 .
\end{aligned}
$$

В дальнейшем мы пренебрегаем ультраффиолетовыми расходимостями в действии и включаем все конечные слагаемые перенормировочного типа, пропорциональные $\int d x V^{d / 2}$, в переопределение $\mu^{2}$.

Аналогично случаю потенциала Коулмена-Вайнберга роль массового параметра перенормировки $\mu^{2}$ состоит в обезразмеривании аргумента второго логарифма в выражении (4.8). Тем не менее обычное выражение (4.7) для потенциала Коулмена-Вайнберга в случае малых $V$ заменяется качественно другой, нелокальной структурой. Для малых потенциалов градиентные слагаемые доминируют, и, таким образом, исходное коулмен-вайнберговское слагаемое не выживает в данном приближении. Однако оно может быть восстановлено при переходе к формальному пределу постоянного потенциала, когда аргумент второго логарифма стремится к $\mu^{2} \int d x V$, а бесконечный объемньй фактор $\int d x$ сокрашается в разности двух логарифмов, так что $\bar{\Gamma} \rightarrow \Gamma_{\mathrm{CW}}, V(x) \rightarrow$ const, где $\Gamma_{\mathrm{CW}}$ есть действие Коулмена-Вайнберга $(2.15)$ в размерности $d=4$. Это подтверждает непротиворечивость используемого приближения. 
Для более высоких (четных) размерностей $d>4$ логарифмическое слагаемоев действии оказывается сублидируюшим [21] и в ответе доминируют следуюшие, не зависяшие от перенормировки, отрицательно определенные слагаемые:

$$
\begin{aligned}
\bar{\Gamma} \simeq & -\frac{1}{(8 \pi)^{d / 2}} \frac{2}{d(d-2)} \int d x V^{2}(x)\left(\frac{\int d y V^{2}(y)}{\int d y V \frac{1}{V-\square} V(y)}\right)^{d / 2-2}+ \\
& +\frac{1}{2(4 \pi)^{d / 2}} \int d x \frac{(-V)^{d / 2}(x)}{(d / 2) !} \ln \left(\frac{\int d y V^{2}(y)}{\int d y V \frac{\mu^{2}}{V-\square} V(y)}\right) .
\end{aligned}
$$

Здесь второе слагаемое для малых, но пространственно неоднородных потенциалов в обшем случае много меньше первого, поскольку в силу оценки (4.7)

$$
\frac{\int d y V^{2}(y)}{\int d y V \frac{1}{V-\square} V(y)} \gg V(x) .
$$

Тем не менее в пределе, когда потенциал стремится к константе, это отношение (которое есть не что иное, как $1 / s_{*}$ ) стремится к $V$, так что оба слагаемых становятся величинами одного порядка. Первое становится слагаемым перенормировочного типа, пропорциональным $\int d x V^{d / 2}$, в то время как второе становится (логарифмически более сильным) $d$-мерным потенциалом Коулмена-Вайнберга (2.15). Так что принцип соответствия выполняется и в высших размерностях.

4.2. Большой потенциал. Другой класс потенциалов, для которых кусочно-гладкое приближение (4.5) эффективно, соответствует противоположной оценке:

$$
V_{0} R^{2} \gg 1
$$

т.е. случаю больших потенциалов в единицах обратного характерного размера носителя. В данном случае градиентные слагаемые уже не доминируют в сравнении с потенциальными слагаемыми и эффективное действие содержит слагаемое типа Коулмена-Вайнберга, модифицированное особой нелокальной поправкой (ранее вьведенной в [14] для $d=4)$,

$$
\bar{\Gamma} \simeq \Gamma_{\mathrm{CW}}+\frac{1}{(4 \pi)^{d / 2}} \frac{2}{d(d-2)} \int_{|x| \leqslant R} d x\langle V \Phi\rangle^{d / 2} .
$$

Эта поправка содержит среднее значение функции $V \Phi(x)$ по компактному носителю

$$
\langle V \Phi\rangle \equiv \int_{|x| \leqslant R} d x V \Phi / \int_{|x| \leqslant R} d x .
$$

Этот алгоритм снова выдерживает проверку в формальном пределе постоянного потенциала, так как в этом пределе функция $\Phi(x)$, определенная в $(3.8)$, стремится к нулю (а размер носителя $R$ стремится к бесконечности). 


\section{5. ВКЛЮЧЕНИЕ ГРАВИТАЦИИ}

Полученная асимптотика позднего времени обладает тем замечательным свойством, что может быть непосредственно обобщена на случай искривленного пространства-времени. В этом случае плоская метрика заменяется на метрику искривленного пространства, а интервал в анзаце (2.1) заменяется на мировую функцию - половину квадрата геодезического расстояния между точками $x$ и $y$,

$$
\delta_{\mu \nu} \rightarrow g_{\mu \nu}(x), \quad \frac{|x-y|^{2}}{2} \rightarrow \sigma(x, y) .
$$

Квазиклассический анзац (2.1) принимает вид

$$
K(s \mid x, y)=\frac{1}{(4 \pi s)^{d / 2}} \exp \left[-\frac{\sigma(x, y)}{2 s}\right] \Omega(s \mid x, y) g^{1 / 2}(y),
$$

где $\Omega(s \mid x, y)$ - бискалярная величина, предел которой при малых временах в отличие от $(2.3)$ есть

$$
\begin{gathered}
\Omega(s \mid x, y) \rightarrow \Delta^{1 / 2}(x, y), \quad s \rightarrow 0, \\
\Delta(x, y)=g^{-1 / 2}(x)\left(\operatorname{det} \partial_{\mu}^{x} \partial_{\nu}^{y} \sigma(x, y)\right) g^{-1 / 2}(y) \neq 0,
\end{gathered}
$$

в терминах детерминанта Паули-Ван Флека-Моретт (веса 0) [16], [17] ${ }^{5}$.

Выделение фактора $\Delta^{1 / 2}(x, y)$ в (5.1) с точки зрения разложения при больших временах излишне. Тем не менее эта величина весьма важна и связана с сушественным предположением, лежашим в основе наших результатов. Мы предполагаем отсутствие так называемых фокальных точек для совокупности геодезических, определяюших мировую функцию $\sigma(x, y)$. Таким образом, мы предполагаем, что $\Delta(x, y) \neq 0$ для всех пар точек $x$ и $y$, что гарантирует глобальную определенность и единственность $\sigma(x, y)$ на рассматриваемом асимптотически плоском пространстве. Данное предположение обосновывает анзац (5.2), который должен быть корректно глобально определен, так как коэффициенты разложения (3.2) будут решениями эллиптической краевой задачи с граничными условиями на бесконечности.

Мы также предполагаем, что метрика является асимптотически плоской и в декартовых координатах обладает следуюшим характером убывания на бесконечности, характерным для $d$-мерного евклидова пространства:

$$
\left.g_{\mu \nu}(x)\right|_{|x| \rightarrow \infty}=\delta_{\mu \nu}+O\left(\frac{1}{|x|^{d-2}}\right) .
$$

Это требование не исключает присутствия каустик у геодезических, $\Delta(x, y)=0$, что зависит от локальных свойств гравитационного поля и не связано с его свойствами на

\footnotetext{
${ }^{5)}$ Мы определяем $\delta(x, y)$-функцию как скаляр по отношению к первому аргументу $x$ и плотность единичного веса по отношению ко второму аргументу $y$. Соответственно ядро теплопроводности имеет такие же веса по своим аргументам. Такая асимметрия по $x$ и $y$ объясняет присутствие фактора $g^{1 / 2}(y)$ в (5.2) и бискалярную природу $\Omega(s \mid x, y)$.
} 
больших расстояниях. Предположение геодезической выпуклости, возможно, является слишком сильным для некоторых физически интересных ситуаций, и мы надеемся, что полученная асимптотика позднего времени останется справедливой даже при наличии каустик (хотя, возможно, ценой дополнительных вкладов, которые мы не рассматриваем в данной статье) ${ }^{6)}$.

5.1. Старший порядок разложения. Повторяя аргументацию раздела 3 , мы немедленно находим последовательность рекуррентных уравнений на коэффициенты $1 / s$ разложения (3.2) для ядра теплопроводности оператора $F(\nabla),(1.4)$, в искривленном пространстве-времени. Их решение в лидируюшем порядке оказывается непосредственной ковариантизацией результата, полученного для плоского пространства-времени (3.10). В ответ входит универсальная скалярная функция $\Phi(x)$, которая теперь является функционалом как от потенциала, так и от метрики,

$$
\Phi(x) \equiv \Phi(x)\left[g_{\mu \nu}, V\right]=1+\int d y G(x, y) V(y),
$$

и выражается через функцию Грина искривленного пространства $G(x, y) \equiv$ $G(x, y)\left[V, g_{\mu \nu}\right]$, удовлетворяюшую тем же граничным условиям на бесконечности:

$$
F(\nabla) G(x, y)=\delta(x, y),\left.\quad G(x, y)\right|_{|x| \rightarrow \infty}=0 .
$$

Таким образом, старший порядок разложения $K(s \mid x, y)$ при больших временах есть ковариантизация результата для плоского пространства-времени. Примечательно, что практически то же самое происходит и в случае функционального следа ядра теплопроводности. Его лидируюшая асимптотика задается двумя слагаемыми. Первое из них есть непосредственная ковариантизация (3.13), а второе выражается через новую структуру - поверхностный интеграл по пространственно-временно́й бесконечности, отражающий тот факт, что метрика пространства-времени является асимптотически плоской:

$$
\begin{aligned}
W_{0} & =-\int d x g^{1 / 2} V \Phi(x)+\frac{1}{6} \Sigma\left[g_{\infty}\right] \\
\Sigma\left[g_{\infty}\right] & =\int_{|x| \rightarrow \infty} d \sigma^{\mu} \delta^{\alpha \beta}\left(\partial_{\alpha} g_{\beta \mu}-\partial_{\mu} g_{\alpha \beta}\right) .
\end{aligned}
$$

Доказательство этого утверждения не может опираться на ковариантное разложение по степеням $V$ и $R_{\mu \nu}[9]$, [11]-[13], [18]. В данном случае, в отличие от случая пертурбативного разложения только по степеням потенциала, $n$-й член разложения по кривизнам

\footnotetext{
6)Эта надежда основана на простом факте, что лидирующий порядок в $1 / s$-разложении не чувствителен к свойствам мировой функции. В следующих порядках в основной интересующий нас объект $\operatorname{Tr} K(s)$ входит предел совпадающих точек мировой функции $\sigma(x, x)=0$, в то время как асимптотические коэффициенты в $\Omega_{n}(x, y)$ нелокально зависят от глобальной геометрии и в них могут возникнуть дополнительные вклады от каустик, аналогичные вкладам в случае, когда точки $x$ и $y$ вне геодезически выпуклой окрестности соединяются несколькими геодезическими [22].
} 
в явном виде неизвестен (даже вычисление третьего порядка является весьма сложной вычислительной задачей [12]). Поэтому остается только метрический аналог вариационного уравнения (3.7) для восстановления функционального следа по ядру $K(s \mid x, y)$. Но даже этого оказывается недостаточно, так как вариационное уравнение (3.7) справедливо только с точностью до поверхностных слагаемых, которые в случае вариации по метрике не обрашаются в нуль. Действительно, вариационное уравнение для экспоненты оператора фактически есть

$$
\begin{aligned}
\delta_{g} \operatorname{Tr} e^{s F}= & \int_{0}^{s} d t \operatorname{Tr}\left[e^{(s-t) F} \delta_{g} F e^{t F}\right]=s \operatorname{Tr}\left[\delta_{g} F e^{s F}\right]+ \\
& + \text { поверхностные слагаемые, }
\end{aligned}
$$

где второе равенство выполняется вследствие свойства цикличности функционального следа. В отличие от конечномерных матрищ в данном случае свойство цикличности бесконечномерного функционального следа основано на неоднократном (в действительности бесконечнократном) интегрировании по частям, таким образом допуская появление конечных поверхностных интегралов. К счастью, для асимптотически плоского пространства-времени их можно вычислить в лидируюшем порядке теории возмушений. Поэтому для вывода (5.7) мы воспользуемся комбинацией вариационной и пертурбативной техник.

Рассмотрим сначала вариационную производную $\operatorname{Tr} K(s)$ по метрике в классе вариаций $\delta g_{\mu \nu}$, достаточно быстро убывающих на бесконечности, так что можно пренебречь вкладом от интегрирования по частям в выражении (5.9). Соответствуюшая вариационная производная принимает вид

$$
\begin{aligned}
\frac{\delta \operatorname{Tr} K(s)}{\delta g_{\mu \nu}(x)} & =\left.s \int d y \frac{\delta F\left(\nabla_{y}\right)}{\delta g_{\mu \nu}(x)} K\left(s \mid y, y^{\prime}\right)\right|_{y^{\prime}=y}= \\
& =-\left.s g^{1 / 2}(x) f^{\mu \nu}\left(\nabla_{x}, \nabla_{y}\right) K(s \mid x, y)\right|_{x=y},
\end{aligned}
$$

где

$$
f^{\mu \nu}\left(\nabla_{x}, \nabla_{y}\right)=-\nabla_{x}^{(\mu} \nabla_{y}^{\nu)}+\frac{1}{2} g^{\mu \nu} \square_{x}+\frac{1}{2} g^{\mu \nu} \nabla_{x}^{\lambda} \nabla_{\lambda}^{y} .
$$

Непосредственная проверка показывает, что в этом классе вариаций, возмушаюших только объемную часть (5.7), равенство (5.10) в старшем порядке по $1 / s$ действительно выполняется [15],

$$
\frac{\delta W_{0}}{\delta g_{\mu \nu}(x)}=-\left.g^{1 / 2}(x) f^{\mu \nu}\left(\nabla_{x}, \nabla_{y}\right) \Phi(x) \Phi(y)\right|_{x=y} .
$$

При вариации функционала $W_{0}$, заданного выражением (5.7), используется выражение для вариации по метрике функции $\Phi(x)$ - аналог (3.11) -

$$
\frac{\delta \Phi(x)}{\delta g_{\mu \nu}(y)}=G(x, y) f^{\mu \nu}\left(\vec{\nabla}_{y}, \overleftarrow{\nabla}_{y}\right) \Phi(y)
$$


где стрелки указывают направление действия производных.

Метрические вариации, убывающие при $|x| \rightarrow \infty$ как поправки от асимптотически плоского пространства-времени $(5.4), \delta g_{\mu \nu}(x) \sim 1 /|x|^{d-2}$, порождают неисчезающие (при устремлении границы к бесконечности) поверхностные вклады в вариационное уравнение (5.9) и при этом не могут быть проверены с помощю (5.10). К счастью, ковариантная теория возмушений, описанная в п. 2.3, показывает [11], что такие слагаемые (возникающие при циклической перестановке ядер теплопроводности под знаком функционального следа) возникают только в линейном порядке пертурбативного разложения по степеням отклонений $h_{\mu \nu}$ метрики $g_{\mu \nu}$ от метрики плоского пространства. Таким образом, они могут быть восстановлены из простого сравнения (5.7) с асимптотикой позднего времени, полученной в [12], [13] до третьего порядка по кривизнам и потенциалу ${ }^{7)}$. Эта асимптотика для оператора вида (1.4) может быть представлена в виде

$$
\begin{aligned}
\operatorname{Tr} K(s)= & -\frac{s}{(4 \pi s)^{d / 2}} \int d x g^{1 / 2}\left\{V+V \frac{1}{\square} V+V \frac{1}{\square} V \frac{1}{\square} V+O\left(V^{4}\right)\right\}+ \\
& +\frac{1}{6} \frac{s}{(4 \pi s)^{d / 2}} \int d x g^{1 / 2}\left\{R-R_{\mu \nu} \frac{1}{\square} R^{\mu \nu}+\frac{1}{2} R \frac{1}{\square} R+\right. \\
& +\frac{1}{2} R\left(\frac{1}{\square} R^{\mu \nu}\right) \frac{1}{\square} R_{\mu \nu}-R^{\mu \nu}\left(\frac{1}{\square} R_{\mu \nu}\right) \frac{1}{\square} R+ \\
& +\left(\frac{1}{\square} R^{\alpha \beta}\right)\left(\nabla_{\alpha} \frac{1}{\square} R\right) \nabla_{\beta} \frac{1}{\square} R-2\left(\nabla^{\mu} \frac{1}{\square} R^{\nu \alpha}\right)\left(\nabla_{\nu} \frac{1}{\square} R_{\mu \alpha}\right) \frac{1}{\square} R- \\
& \left.-2\left(\frac{1}{\square} R^{\mu \nu}\right)\left(\nabla_{\mu} \frac{1}{\square} R^{\alpha \beta}\right) \nabla_{\nu} \frac{1}{\square} R_{\alpha \beta}+\mathrm{O}\left[R_{\mu \nu}^{4}\right]\right\} .
\end{aligned}
$$

Нелокальное разложение $\Phi(x)$,

$$
\Phi(x)=1+\frac{1}{\square} V(x)+\frac{1}{\square} V \frac{1}{\square} V(x)+O\left(V^{3}\right),
$$

очевидно восстанавливает из первого интеграла в (5.14) первое слагаемое в (5.7), содержащее явно только степени потенциала с зависящими от метрики нелокальностями.

Второй интеграл в (5.14) является топологическим инвариантом, не зависящим от локальных вариаций метрики во внутренней области пространства-времени; именно в этом классе вариаций $\delta g_{\mu \nu}(x)$ ранее была вычислена функциональная производная (5.10). Непосредственное разложение по степеням $h_{\mu \nu}, g_{\mu \nu}=\delta_{\mu \nu}+h_{\mu \nu}$, на плоском фоне в декартовых координатах показывает, что этот член сводится к поверхностному интегралу на бесконечности. Для класса асимптотически плоских метрик с $h_{\mu \nu}(x) \sim$ $1 /|x|^{d-2},|x| \rightarrow \infty$, этот поверхностный интеграл линеен по возмушениям (вклады более высоких степеней $h_{\mu \nu}$ в этот интеграл исчезают) и содержит только локальньй вклад

\footnotetext{
7) Этого приближения достаточно для проверки вклада, линейного по $h_{\mu \nu}$. В ковариантной теории возмущений возмущение метрики раскладывается в бесконечный ряд по кривизнам, что является платой за общую ковариантность разложения.
} 
асимптотики на бесконечности, $g_{\mu \nu}^{\infty}(x)=\delta_{\mu \nu}+\left.h_{\mu \nu}(x)\right|_{|x| \rightarrow \infty}$,

$$
\begin{aligned}
\int d x & g^{1 / 2}\left\{R-R_{\mu \nu} \frac{1}{\square} R^{\mu \nu}+\frac{1}{2} R \frac{1}{\square} R+\right. \\
& +\frac{1}{2} R\left(\frac{1}{\square} R^{\mu \nu}\right) \frac{1}{\square} R_{\mu \nu}-R^{\mu \nu}\left(\frac{1}{\square} R_{\mu \nu}\right) \frac{1}{\square} R+ \\
& +\left(\frac{1}{\square} R^{\alpha \beta}\right)\left(\nabla_{\alpha} \frac{1}{\square} R\right) \nabla_{\beta} \frac{1}{\square} R-2\left(\nabla^{\mu} \frac{1}{\square} R^{\nu \alpha}\right)\left(\nabla_{\nu} \frac{1}{\square} R_{\mu \alpha}\right) \frac{1}{\square} R- \\
& -2\left(\frac{1}{\square} R^{\mu \nu}\right)\left(\nabla_{\mu} \frac{1}{\square} R^{\alpha \beta}\right) \nabla_{\nu} \frac{1}{\square} R_{\alpha \beta}+\mathrm{O}\left[R_{\mu \nu}^{4}\right\}= \\
= & \int_{|x| \rightarrow \infty} d \sigma^{\mu}\left(\partial^{\nu} h_{\mu \nu}-\partial_{\mu} h\right) \equiv \Sigma\left[g_{\infty}\right] .
\end{aligned}
$$

Здесь $d \sigma^{\mu}$ - мера объема сферы радиуса $|x| \rightarrow \infty, \partial^{\mu}=\delta^{\mu \nu} \partial_{\nu}$, a $h=\delta^{\mu \nu} h_{\mu \nu}$.

Ковариантный способ проверки данного соотношения заключается в вычислении вариации этого интеграла по метрике и демонстрации того, что подынтегральное выражение является полной дивергенцией, которая дает ожидаемое поверхностное слагаемое, линейное по $\delta g_{\mu \nu}(x)=h_{\mu \nu}(x)$ [21]. Таким образом, корректное выражение для $W_{0}$ модифицируется поверхностным слагаемым $\Sigma\left[g_{\infty}\right]$, не дающим вклада в вариационную производную $\delta W_{0} / \delta g_{\mu \nu}(x)$ при конечных $|x|$.

Для асимптотически плоских метрик с законом убывания на бесконечности $h_{\mu \nu}(x) \sim$ $M /|x|^{d-2},|x| \rightarrow \infty$, поверхностный интеграл $\Sigma\left[g_{\infty}\right]$ присутствует в действии Гильберта-Эйнштейна

$$
S_{\mathrm{HE}}[g] \equiv-\int d x g^{1 / 2} R(g)+\Sigma\left[g_{\infty}\right]
$$

обеспечивая корректность вариационной процедуры, приводяшей к уравнениям Эйнштейна. Этот поверхностный интеграл может быть переписан ковариантно в форме Гиббонса-Хокинга $S_{\mathrm{GH}}[g]=\Sigma\left[g_{\infty}\right]$ как поверхностный интеграл от удвоенного следа внешней кривизны $K$ границы (с учетом соответствуюшего вычитания бесконечного вклада фонового плоского пространства-времени) [23]. Таким образом, (5.16) - это поверхностный интеграл, локально зависящий от метрики на границе и ее нормальных производных. Ценность соотношения (5.16) заключается в выражении поверхностного интеграла в виде объемного интеграла по пространству-времени от нелокального функционала метрики в объеме. Последний не содержит явно дополнительных структур типа векторного поля, ортогонального к границе, хотя эти структуры неявно присутствуют в граничных условиях для нелокальных операций в подынтегральном выражении (5.16).

Попутно заметим, что равенство (5.16) может быть использовано для записи действия Гильберта-Эйнштейна (в евклидовом пространстве) (5.17) в виде нелокального разложения по кривизнам, начинающегося с квадратичного порядка по кривизне. Это наблюдение служит основой для последовательных ковариантных нелокальных модификаций эйнштейновской теории [10], мотивированных проблемами космологической постоянной и космологического ускорения [24]. 
5.2. Конформные свойства. Важный случай зависящего от метрики потенциала в (1.4) соответствует оператору конформного скалярного поля. Для случая размерности $d$ он имеет вид

$$
F(\nabla)=\square-\frac{1}{4} \frac{d-2}{d-1} R
$$

При локальных конформных (вейлевских) преобразованиях метрики, стремящихся к единице на бесконечности, этот оператор преобразуется однородно, домножаясь слева и справа на соответствующие степени конформного фактора:

$$
\begin{gathered}
\bar{g}_{\mu \nu}(x)=\Psi^{4 /(d-2)}(x) g_{\mu \nu}(x), \quad \Psi(x)=1+O\left(\frac{1}{|x|^{d-2}}\right), \quad|x| \rightarrow \infty, \\
\bar{F}(\nabla)=\Psi^{-1-4 /(d-2)}(x) F(\nabla) \Psi(x)
\end{gathered}
$$

В силу этого свойства универсальная функция $\Phi(x)(5.5)$ также преобразуется однородно, так как она удовлетворяет однородному уравнению для оператора (5.18) с теми же единичными граничными условиями,

$$
\bar{\Phi}(x) \equiv \Phi(x)\left[\bar{g}_{\mu \nu}\right]=\frac{\Phi(x)}{\Psi(x)} .
$$

Еще одно интересное свойство - конформное преобразование скалярной кривизны:

$$
\bar{R}(x)=-4 \frac{d-1}{d-2} \Psi^{-1-4 /(d-2)}(x)[F(\nabla) \Psi(x)],
$$

которое означает, что $\Phi(x)$ может рассматриваться как специальный случай конформного преобразования к конформной калибровке нулевой скалярной кривизны, $\bar{R}=0$, когда параметр $\Psi$ этого преобразования равен $\Phi$.

Рассмотрим теперь объемную часть лидирующей асимптотики $W_{0}(5.7)$ для случая конформного оператора (5.18)

$$
W_{0}[g]=-\frac{1}{4} \frac{d-2}{d-1} \int d x g^{1 / 2} R \Phi+\frac{1}{6} \Sigma\left[g_{\infty}\right] .
$$

В силу (5.22) она преобразуется следующим образом:

$$
-\frac{1}{4} \frac{d-2}{d-1} \int d x \bar{g}^{1 / 2} \bar{R} \bar{\Phi}=\int d x g^{1 / 2} \Phi F(\nabla) \Psi=\int_{\infty} d \sigma^{\mu}\left(\partial_{\mu} \Psi-\partial_{\mu} \Phi\right) .
$$

Здесь использовалось уравнение $F(\nabla) \Phi=0$, производилось интегрирование по частям и учитьвалось, что $\Phi$ и $\Psi$ равны 1 на бесконечности (но их производные в общем случае стремятся к нулю недостаточно быстро, чтобы можно было пренебречь поверхностным интегралом). Второе слагаемое в поверхностном интеграле может быть преобразовано обратно к объемному виду (теперь не содержащему $\Psi$ ):

$$
-\int_{\infty} d \sigma^{\mu} \partial_{\mu} \Phi=-\int d x g^{1 / 2} \square \Phi=-\frac{1}{4} \frac{d-2}{d-1} \int d x g^{1 / 2} R \Phi,
$$


так что

$$
\int d x \bar{g}^{1 / 2} \bar{R} \bar{\Phi}=\int d x g^{1 / 2} R \Phi-4 \frac{d-1}{d-2} \int_{\infty} d \sigma^{\mu} \partial_{\mu} \Psi
$$

С учетом конформного преобразования интеграла Гиббонса-Хокинга (5.8),

$$
\bar{\Sigma} \equiv \Sigma\left[\bar{g}_{\infty}\right]=\Sigma-4 \frac{d-1}{d-2} \int_{\infty} d \sigma^{\mu} \partial_{\mu} \Psi
$$

это означает, что следующая линейная комбинация:

$$
S_{\text {conf }}[g]=-\int d x g^{1 / 2} R \Phi+\Sigma\left[g_{\infty}\right]
$$

конформно-инвариантна в классе локальных вейлевских преобразований (5.19),

$$
S_{\text {conf }}[g]=S_{\text {conf }}[\bar{g}]
$$

В точности такая же структура может быть получена из действия (5.17) с помошью перехода к конформной калибровке, обращающей в нуль скалярную кривизну. Осуществив преобразование (5.19) с $\Psi=\Phi[g]$, находим, что

$$
S_{\mathrm{conf}}\left[g_{\mu \nu}\right]=S_{\mathrm{HE}}\left[g_{\mu \nu} \Phi^{4 /(d-2)}[g]\right]
$$

Конформная инвариантность этого выражения очевидна, так как $g_{\mu \nu} \Phi^{4 /(d-2)}[g]-$ конформный инвариант для преобразования (5.19). Фактически этот функционал в 4-мерном контексте был предложен в [25] как конформное расширение вне массовой оболочки теории Эйнштейна в вакууме (или в присутствии бесследовых материальных источников).

Рассмотрим теперь конформные свойства асимптотики ядра теплопроводности для оператора (5.18). Для самого ядра теплопроводности эти свойства напрямую следуют из закона преобразования функции $\Phi(x)(5.21)$, что в лидируюшем порядке дает

$$
\bar{\Omega}(x, y) \bar{g}^{1 / 2}(y)=\Psi^{-1}(x)\left[\Omega(x, y) g^{1 / 2}(y)\right] \Psi^{1+4 /(d-2)}(y)
$$

Это преобразование похоже на преобразование оператора (5.20). Тем не менее такое совпадение случайно, потому что в отличие от (5.20) ядро теплопроводности не преобразуется простым однородным образом. Уравнение (5.20) не есть преобразование подобия множители слева и справа от $F(\nabla)$ в (5.20) не обратны друг другу. Дополнительный фактор $\Psi^{-4 /(d-2)}$ в $\bar{F}(\nabla)=\Psi^{-1}\left(\Psi^{-4 /(d-2)} F(\nabla)\right) \Psi$ означает, что оператор $\bar{F}(\nabla)$ подобен другому оператору $-\Psi^{-4 /(d-2)} F(\nabla)$, имеющему другое ядро теплопроводности. На самом деле этот фактор, определяющий конформное преобразование метрики (5.19), приводит к точно вычисляемой конформной аномалии эффективного действия. Но для 
самого ядра теплопроводности и его функционального следа он порож дает нетривиальньй закон преобразования. Для функционального следа это преобразование с инфинитезимальным $\delta \Psi(x)$ в $\Psi(x)=1+\delta \Psi(x)$ выглядит так:

$$
\begin{aligned}
\left.\delta_{\Psi} \operatorname{Tr} \bar{K}(s)\right|_{\Psi=1} & =-\frac{4}{d-2} s \frac{d}{d s} \operatorname{Tr}(\delta \Psi K(s))= \\
& =-\frac{4}{d-2} s \frac{d}{d s} \int d x \delta \Psi(x) \Omega(s \mid x, x) .
\end{aligned}
$$

Когда вариация $\delta \Psi(x)$ сосредоточена на компактном носителе, мы вправе использовать лидирующую асимптотику $\Omega(s \mid x, x) \sim \Phi^{2}(x) /(4 \pi s)^{d / 2}$, так как в этом случае область $|x| \rightarrow \infty$ (в которой нарушается равномерность этой асимптотики) не входит в область интегрирования и $\delta_{\Psi} \operatorname{Tr} K(s)=O\left(1 / s^{d / 2}\right)$ при $s \rightarrow \infty$. Поэтому лидируюшая асимптотика $W_{0}$ является конформным инвариантом относительно вейлевских преобразований с компактным носителем. Действительно, оба члена в (5.23) инвариантны по отдельности в силу (5.26) и (5.27).

Для конформных преобразований более обшего вида с $\delta \Psi(x) \rightarrow 0$, но $\delta \Psi(x) \neq 0$, при $|x| \rightarrow \infty$ аналогичные заключения не могут быть сделаны на основании (5.32), так как теперь появляются области, где неравномерная асимптотика $\Omega(s \mid x, x)$ нарушается. Поэтому для такого класса преобразований функционал $W_{0}$ не обязательно будет конформно-инвариантным, что объясняет несоответствие коэффициентов объемного и поверхностного членов в (5.23) по сравнению со случаем конформного расширения теории Гильберта-Эйнштейна (5.28).

Довольно интересно, что в исключительном случае 4-мерного пространства-времени инвариантность $W_{0}$ сохраняется даже для преобразований с некомпактным носителем. В этом случае коэффициент неминимального взаимодействия скалярного поля с кривизной $(d-2) / 4(d-1)=1 / 6$ и

$$
W_{0}[g]=\frac{1}{6} S_{\text {conf }}[g], \quad d=4
$$

5.3. Проблемы с сублидирующим порядком. Как мы увидим, учет гравитации приводит к ряду проблем в высших порядках асимптотики позднего времени. В частности, в сублидируюшем порядке уравнение для $\Omega_{1}(x, y)$, обобшаюшее $(3.13)$ на случай искривленного пространства-времени, записывается в виде

$$
F(\nabla) \Omega_{1}(x, y)=\left(\sigma^{\mu}(x, y) \nabla_{\mu}+\frac{1}{2} \square \sigma(x, y)-\frac{d}{2}\right) \Phi(x) \Phi(y)
$$

и обладает симметричным решением, аналогичным (3.14) [15],

$$
\begin{aligned}
\Omega_{1}(x, y)= & \frac{1}{2} \psi(x, y)+\frac{1}{2} \psi(y, x)- \\
& -\frac{1}{2} \frac{1}{F\left(\nabla_{x}\right)} \vec{F}\left(\nabla_{x}\right)[\Phi(x) \sigma(x, y) \Phi(y)] \overleftarrow{F}\left(\nabla_{y}\right) \frac{\overleftarrow{1}}{F\left(\nabla_{y}\right)}
\end{aligned}
$$


Здесь $\psi(x, y)$ - специальная двухточечная функция:

$$
\psi(x, y)=\frac{1}{F\left(\nabla_{x}\right)}\left[2 \sigma^{\mu}(x, y) \nabla_{\mu} \Phi(x)+(\square \sigma(x, y)-d) \Phi(x)\right] \Phi(y),
$$

а стрелки указывают направление действия дифференциальных операторов и функций Грина, $1 / F(\nabla)$, записанных в операторном виде. Это, естественно, подразумевает, что интегрирование по частям, которое обратило бы действие $F(\nabla)$ на $1 / F(\nabla)$ (и, таким образом, привело бы к полному сокрашению соответствуюших нелокальностей), невозможно без возникновения нетривиальных поверхностных членов. Как показано в [15], для такого $\Omega_{1}(x, y)$ вариационное уравнение для $W_{1}$

$$
\frac{\delta W_{1}}{\delta V(x)}=-g^{1 / 2}(x) \Omega_{1}(x, x)
$$

имеет следующее формальное решение в терминах функции Грина оператора $F(\nabla)$ :

$W_{1}\left[V, g_{\mu \nu}\right]=\left.\frac{1}{2} \int d x g^{1 / 2}(x) \frac{1}{F\left(\nabla_{x}\right)} \vec{F}\left(\nabla_{x}\right)[\Phi(x) \sigma(x, y) \Phi(y)] \overleftrightarrow{F}\left(\nabla_{y}\right)\right|_{y=x}+W_{1}^{\text {metric }}\left[g_{\mu \nu}\right]$.

В этой формуле $W_{1}^{\text {metric }}\left[g_{\mu \nu}\right]$ - некоторый чисто метрический функционал - функциональная "константа" интегрирования уравнения (5.37). Последняя может быть определена только из метрического вариационного уравнения (5.10). Вычисления метрической вариации, проведенные в [21], показывают, что $W_{1}^{\text {metric }}$ - не зависящая от метрики константа: $W_{1}^{\text {metric }}\left[g_{\mu \nu}\right]=$ const.

Этот вывод выглядит антиинтуитивным, поскольку он приводит к очевидному противоречию между (3.15) и плоским пределом (5.38), заключающемуся в первом слагаемом выражения (3.15). Это слагаемое отсутствует в (5.38), и попытка отождествить его с константой,

$$
W_{1}^{\text {metric }}=\int d x \times 1,
$$

противоречит процедуре ковариантизации при переходе к искривленному пространству (по сушеству, установленной в рамках ковариантного разложения по кривизнам [11], [12]):

$$
\operatorname{Tr} K(s)=\frac{1}{(4 \pi s)^{d / 2}} \int d x \times 1+\cdots \rightarrow \frac{1}{(4 \pi s)^{d / 2}} \int d x g^{1 / 2}(x) \times 1+\cdots .
$$

Причиной этого несоответствия является то, что, к сожалению, алгоритмы (5.35), (5.36) и (5.38), строго говоря, работают только в плоском пространстве. Основная сложность заключается в том, что нелокальная функция $\psi(x, y)$ хорошо (и однозначно) определена, только когда выражение в квадратных скобках в (5.36) достаточно быстро убывает на бесконечности. Первое слагаемое этого выражения убывает степенным образом $1 /|x|^{d-2}$, так как $\sigma^{\mu}(x, y) \sim|x|$ и $\nabla_{\mu} \Phi(x) \sim 1 /|x|^{d-1}$, и поэтому хорошо определено, по меньшей мере для размерностей $d>4$. Наоборот, второе слагаемое пропорционально 
девиации геодезических, $\square \sigma(x, y)-d$, которая имеет следующее весьма умеренное убывание на бесконечности:

$$
\square \sigma(x, y)-d \sim 1 /|x|, \quad|x| \rightarrow \infty
$$

Это приводит к квадратичной расходимости чисто метрического вклада в (2.12) в инфракрасном пределе ${ }^{8)}$.

Проследив в обратном порядке происхождение проблемы вплоть до уравнения (5.34), можно заметить, что источник в правой части этого уравнения ведет себя как $O(1 /|x|)$ при $|x| \rightarrow \infty$, так что решение $\Omega_{1}(x, y) \sim|x|$ оказывается неубываюшим на бесконечности и поэтому не фиксируется условиями типа Дирихле. Следует ожидать, что фиксация этого произвола приведет к регуляризации интеграла в $\psi(x, y)$ и однозначно определит все величины в сублидируюшем порядке, но это пока остается задачей на будущее.

Вывод выражения (5.38) основан на операциях с нерегуляризованными расходящимися интегралами, что порождает серьезные сомнения в его достоверности. Однако довольно тонкая проверка метрической вариации [21] наводит на мысль, что при некоторой процедуре регуляризации инфракрасных расходимостей в (5.38) и (5.39) алгоритмы сохранятся также за рамками плоского пространства. Совместность этих алгоритмов с ковариантной теорией возмущений [11], [12], нетривиальная ввиду этих расходимостей, должна быть основана на выделении ковариантного интеграла $\int d x g^{1 / 2} \times 1$ (который сам по себе расходится!) из расходяшихся нелокальных выражений (5.38), (5.39). Инфракрасная расходимость $\int d x g^{1 / 2} \times 1$ отражает непрерывность спектра оператора $F(\nabla)$ и в плоском пределе представляет собой тривиальную константу, не влияющую на физические предсказания. В искривленном пространстве этот интеграл становится функционально зависяшим от геометрии и поэтому включает космологический член. Таким образом, сублидируюший порядок разложения позднего времени оказывается связанным с проблемой космологической постоянной, что мы кратко обсудим в заключении.

\section{6. ЗАКЛЮЧЕНИЕ}

Основные результаты, представленные выше, включают в себя непертурбативную асимптотику ядра теплопроводности (3.10) и (3.12), ее обобщение на случай искривленного пространства-времени (5.7), (5.8), а также соответствуюшие новые алгоритмы вычисления нелокального эффективного действия (4.8), (4.9) и (4.12). Обсудим возможные обобшения и приложения этих результатов, а также остающиеся пробелы в использованной технике.

Непертурбативное эффективное действие, альтернативное потенциалу КоулменаВайнберга, весьма интересно в связи с рядом приложений. С первого взгляда видно,

\footnotetext{
8) Предел плоского пространства, наоборот, хорошо определен, потому что бискаляр геодезического расхождения (5.41) тождественно обращается в нуль для $\sigma(x, y)=|x-y|^{2} / 2$. Следовательно, в квадратных скобках выражения (5.38) выживает только слагаемое, содержащее $\nabla_{x}^{\mu} \nabla_{y}^{\nu} \sigma(x, y)=$ $-\delta^{\mu \nu}$, так что $\Omega_{1}(x, y)$ и $W_{1}$ сводятся к выражению (3.14) и второму слагаемому в $(3.15)$, соответственно.
} 
что в алгоритмах (4.8) и (4.9) проявляется нетривиальная и качественно важная зависимость от размерности пространства-времени. Так, для $d>4$ в действие вносят доминирующий вклад не зависящие от перенормировки структуры, в которые не входит перенормировочный параметр $\mu^{2}$. В этом заключается сушественное отличие от ситуации в четырехмерии (4.8), где эффекты инфракрасной области лишь “делокализуют” потенциал Коулмена-Вайнберга, сохраняя доминирующую зависимость от ультрафиолетового масштабного параметра. Это значит, что масштабное поведение не в состоянии полностью определить доминируюший инфракрасный вклад. Также интересно, что лидируюший вклад в старших размерностях отрицательно определен, что, возможно, имеет нетривиальные следствия. В то же время непертурбативные вклады заслуживают дальнейшего анализа, уточняющего их область применимости и учитывающего поправки от факторов, содержащих локальные производные, которыми мы пренебрегали ранее (члены с $\tilde{a}_{n}(x, x)$ при $\left.n>0\right)$.

Что касается общего формализма асимптотики позднего времени, следует подчеркнуть, что трудности, с которыми мы столкнулись в ее высших порядках, представляют серьезную проблему, которую еше предстоит разрешить. Сублидирующий порядок на плоском фоне не страдает от этой проблемы, поскольку выражения (3.14) и (3.15) хорошо определены, но включение гравитации приводит к обсуждаемой проблеме. Тем не менее уже этот порядок по $1 / s$-разложению представляет огромный интерес, так как затрагивает проблему космологической постоянной. В самом деле, космологический член появляется в интеграле (1.8) из (ковариантизации) единичного слагаемого в $\operatorname{Tr} K(s)(5.40)$ как

$$
\Gamma_{\Lambda}=\int d x g^{1 / 2} \Lambda_{\infty}, \quad \Lambda_{\infty}=-\frac{1}{2(4 \pi)^{d / 2}} \int_{0}^{\infty} \frac{d s}{s^{1+d / 2}} \times 1
$$

Космологическая постоянная $\Lambda_{\infty}$ ультрафиолетово расходится, а сам интеграл инфракрасно расходится в координатном смысле ${ }^{9)}$ - объемный интеграл $\int d x g^{1 / 2}$ для асимптотически плоского пространства расходится при $|x| \rightarrow \infty$.

Итак, проблема ковариантизации функционала $W_{1}$ с не зависяшим от метрики слагаемым $W_{1}^{\text {metric }},(5.38)$ и (5.39), равнозначна задаче корректного восстановления ковариантного космологического члена из нелокальных выражений. Чтобы упростить обсуждение этого момента, рассмотрим чисто метрический случай, когда потенциал равен нулю, $V=0, \Phi=1$. Тогда сублидирующий вклад в функциональный след ядра

\footnotetext{
9) Конечно, появление расходимостей указывает на то, что космологическая постоянная не может согласованно появиться в асимптотически плоском пространстве-времени. Вклад (6.1) в безмассовых теориях не несет осмысленной физической информации и сокращается в силу ряда взаимосвязанных механизмов. Сокращение гарантируется вкладом в эффективное действие локальной меры в функциональном интеграле, которая сокращает объемные расходимости [26]. Еще один механизм основан на использовании размерной регуляризации, которая обращает в нуль степенные расходимости. Все эти приемы перестают, однако, работать для массивных теорий или для теорий со спонтанно нарушенной симметрией, где индуцированная вакуумная энергия порождает проблему иерархии [27].
} 
теплопроводности выглядит следуюшим образом:

$$
W_{1}=\frac{1}{2} \int d x d y g^{1 / 2}(x) G(x, y) \square_{x} \square_{y} \sigma(x, y)+\int d x \times 1 .
$$

Первый интеграл, очевидно, обратится в нуль в случае плоского пространства-времени, где $\square_{x} \square_{y} \sigma(x, y)=0$, так что его разложение по степеням кривизны начнется с первого порядка. Этот интеграл инфракрасно расходится и, более того, он расходится квадратично, как и слагаемое первого порядка в разложении по степеням возмушений $h_{\mu \nu}$ метрики (или по кривизнам),

$$
\int d x g^{1 / 2}(x)=\int d x\left(1+\frac{1}{2} h+\cdots\right)=\int d x\left(1-\frac{1}{\square} R(x)+\cdots\right) .
$$

Сравнение (6.3) с (6.2) подсказывает, что первый интеграл в (6.2) начинается с того же нелокального слагаемого, линейного по $(1 / \square) R$ (или локального по $h$ ). Таким образом, полный космологический член кажется закамуфлированным в выражении (6.2) как часть специального нелокального функционала ${ }^{10)}$,

$$
W_{1}=\int d x g^{1 / 2} \times 1+O\left(R^{2}\right)
$$

Данная ситуация не является совершенно новой. Мы уже видели, что локальный поверхностный интеграл Гиббонса-Хокинга имеет нелокальное представление (5.16), которое, в свою очередь, лежит в основе нелокального представления действия Гильберта-Эйнштейна, предложенного в [10]. Тем не менее эта аналогия не годится, в силу того что соответствуюшие интегралы в (6.2) и (6.3) инфракрасно расходятся и требуют специальной регуляризации для их сравнения. Эта регуляризация, по-видимому, будет частью общей схемы, необходимой для придания точного смысла сублидирующему и, возможно, более высоким порядкам $1 / s$-разложения. Введение этой регуляризации может привести к двум возможным сценариям - уравнение (6.4) либо подтвердится, либо окажется, что космологическая постоянная $\int d x g^{1 / 2}$ входит в $\operatorname{Tr} K(s)$ при $s \rightarrow 0$ и при $s \rightarrow \infty$ с различными коэффициентными функциями $s$.

Второй вариант кажется маловероятным, но если он все же реализуется, это будет означать появление нового механизма индуцирования космологического члена. В самом деле, в этом случае зависяшая от $s$ (или парциальная) "космологическая постоянная" $\lambda(s)$ в $\operatorname{Tr} K(s), \quad \operatorname{Tr} K(s)=\lambda(s) \int d x g^{1 / 2}+\cdots$, будет интерполяцией между $\lambda_{<}(s)=$ $1 /(4 \pi s)^{d / 2}$ при $s \rightarrow 0$ и некоторой другой функцией $\lambda_{>}(s)$ при $s \rightarrow \infty$. Все это может породить индуцированную космологическую постоянную

$$
\Lambda_{\text {ind }}=-\frac{1}{2} \int_{0}^{\infty} \frac{d s}{s} \lambda(s) \times 1 \neq 0
$$

\footnotetext{
10) Идея нелокального представления космологического члена ранее рассматривалась в [10], [28]
} 
которая будет определенно не равна нулю, так как в отличие от (6.1) этот интеграл больше не представляет собой чистую степенную расходимость, которая уничтожается размерной регуляризацией. Можно ожидать, что это прояснит интересную связь между проблемой космологической постоянной и инфракрасной асимптотикой ядра теплопроводности и нелокального эффективного действия.

В заключение отметим ряд возможных обобщений предлагаемой техники позднего времени. После преодоления проблем с инфракрасным поведением сублидирующего порядка алгоритмы эффективного действия (4.8) и (4.9) могут быть обобщены на случай искривленного пространства. Это обобшение интересно тем, что может описывать инфракрасные модификации теории гравитации с нелокальной гравитационной “постоянной”, зависяшей от масштаба [10], [24]. Другое интересное обобшение заключается в отказе от ограничения только асимптотически плоскими геометриями. Первое, что предстоит сделать на этом пути, - рассмотреть асимптотически деситтеровские граничные условия, вызывающие особый интерес в связи с явлением космологического ускорения. Это обобщение подразумевает сушественную модификацию как пертурбативной, так и непертурбативной техники вычисления ядра теплопроводности и обобщение гиббонс-хокинговского члена на асимптотически деситтеровское пространство. Еще одно обобщение касается полей более высоких спинов с ковариантными производными, включающими не только метрическую, но также и калибровочные связности, что позволит распространить данную технику на максимально широкий круг физических моделей.

Благодарности. Работа А. О. Барвинского над данной статьей была осушествлена при поддержке РФФИ (грант № 02-01-00930) и Программы поддержки ведущих научных школ (грант № НШ-1578.2003.2). Д. В. Нестеров выражает благодарность за поддержку РФФИ (грант № 05-02-17661), Фонду Ландау (Германия) и Фонду “Династия" (Россия).

\section{Список литературы}

[1] H. van Damm, M. J. Veltman. Nucl. Phys. D. 1970. V. 22. Р. 397; В. И. Захаров. Письма в ЖЭТФ. 1970. Т. 12. № 9. С. 447; A. I. Vainstein. Phys. Lett. 1972. V. 39. P. 393.

[2] M. A. Luty, M. Porrati, R. Rattazzi. JHEP. 2003. V. 0309. P. 029; hep-th/0303116; V. A. Rubakov. Strong coupling in brane-induced gravity in five dimensions. hep-th/0303125.

[3] V. F. Mukhanov, A. Wipf, A. I. Zelnikov. Phys. Lett. B. 1994. V. 332. P. 283.

[4] A. G. Mirzabekian, G. A. Vilkovisky. Class. Quantum Gravit. 1995. V. 12. P. 2173; hep-th/9504028; Ann. Phys. 1998. V. 270. P. 391; gr-qc/9803006.

[5] N. C. Tsamis, R. P. Woodard. Nucl. Phys. 1996. V. 474. P. 235; hep-th/9602315; Ann. Phys. 1997. V. 253. P. 1.

[6] R. Gregory, V. A. Rubakov, S. M. Sibiryakov. Phys. Rev. Lett. 2000. V. 84. P. 5928; hep-th/0002072.

[7] G. R. Dvali, G. Gabadadze, M. Porrati. Phys. Lett. B. 2000. V. 485. P. 208.

[8] M. E. Soussa, R. P. Woodard. Class. Quantum Gravit. 2003. V. 20. P. 2737; astro-ph/0302030.

[9] A. O. Barvinsky, G. A. Vilkovisky. Nucl. Phys. B. 1987. V. 282. P. 163.

[10] A. O. Barvinsky. Phys. Lett. B. 2003. V. 572. P. 109; hep-th/0304229.

[11] A. O. Barvinsky, G. A. Vilkovisky. Nucl. Phys. B. 1990. V. 333. P. 471. 
[12] A. O. Barvinsky, Yu. V. Gusev, G. A. Vilkovisky, V. V. Zhytnikov. Covariant Perturbation Theory (IV). Third Order in the Curvature, Report of the University of Manitoba. Preprint. Winnipeg: Univ. Manitoba, 1993.

[13] A. O. Barvinsky, Yu. V. Gusev, G. A. Vilkovisky, V. V. Zhytnikov. J. Math. Phys. 1994. V. 35. P. 3543.

[14] A. O. Barvinsky, V. F. Mukhanov. Phys. Rev. D. 2002. V. 66. P. 065007; hep-th/0203132.

[15] A. O. Barvinsky, Yu. V. Gusev, V. F. Mukhanov, D. V. Nesterov. Phys. Rev. D. 2003. V. 68. P. 105003; hep-th/0306052.

[16] A. O. Barvinsky, G. A. Vilkovisky. Phys. Rep. 1985. V. 119. P. 1.

[17] B. S. DeWitt. Dynamical Theory of Groups and Fields. N.Y.: Gordon and Breach, 1965.

[18] A. O. Barvinsky, Yu. V. Gusev, G. A. Vilkovisky, V. V. Zhytnikov. J. Math. Phys. 1994. V. 35. P. 3525.

[19] V. P. Frolov, G. A. Vilkovisky. Phys. Lett. B. 1981. V. 106. P. 307; Spherically-symmetric collapse in quantum gravity. In: Quantum Gravity. Proc. of the Second Seminar on Quantum Gravity (Moscow, 1981). Eds. M. A. Markov, P. C. West. N.Y.: Plenum, 1984. P. 267.

[20] A. M. Polyakov. Phys. Lett. B. 1981. V. 103. P. 207.

[21] A. O. Barvinsky, D. V. Nesterov. Nonperturbative heat kernel and nonlocal effective action. Preprint ESI-1444. Vienna: ESI, 2003; hep-th/0402043.

[22] R. Camporesi. Phys. Rep. 1990. V. 196. P. 1.

[23] G. W. Gibbons, S. W. Hawking. Phys. Rev. D. 1977. V. 15. P. 2752.

[24] N. Arkani-Hamed, S. Dimopoulos, G. Dvali, G. Gabadadze. Nonlocal modification of gravity and the cosmological constant problem. hep-th/0209227.

[25] E. S. Fradkin, G. A. Vilkovisky. Phys. Lett. B. 1978. V. 73. P. 209.

[26] E. S. Fradkin, G. A. Vilkovisky. Phys. Rev. D. 1973. V. 8. P. 4241.

[27] S. Weinberg. Rev. Mod. Phys. 1989. V. 61. P. 1.

[28] E. V. Gorbar, I. L. Shapiro. JHEP. 2003. V. 0302. P. 021.

Поступила в редакцию 2.XI.2004 г. 